\title{
HIGHLIGHTS
}

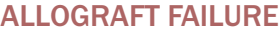

\section{Histological predictors of late renal graft loss}

Long-term renal allograft failure can be predicted by a combination of positive peritubular immunostaining for $\mathrm{C} 4 \mathrm{~d}-\mathrm{a}$ complement degradation product-and evidence of transplant glomerulopathy in biopsy samples, according to a recent study by Kieran and colleagues. "C4d positivity [is] often associated with an active rejection process," reflect the authors. "However, when C4d positivity is associated with [transplant glomerulopathy], the risk [of] graft failure is high."

\section{4 ...C4d positivity alone was not an independent predictor of graft failure $\mathbf{7 7}$}

The investigators identified 99 patients with renal allografts who had undergone biopsy to assess renal dysfunction at least 10 years after transplantation. Positive peritubular C4d immunostaining was observed in biopsy samples from 25 patients, and the histologic features of these samples were compared with those of C4d-negative biopsy tissue using the 2007 Banff renal pathology classification. The researchers found that positive peritubular C4d staining was associated with increased inflammation, increased likelihood of transplant glomerulopathy and an enhanced probability of acute cellular rejection; however, $\mathrm{C} 4 \mathrm{~d}$ positivity alone was not an independent predictor of graft failure.

Crucially, the mean Banff chronic sum score-a composite of Banff indices for transplant glomerulopathy, arterial intimal fibrosis, tubular atrophy and interstitial fibrosis-was significantly higher in C4d-positive biopsies than in C4d-negative biopsies ( $6.36 \pm 0.73$ versus $4.59 \pm 0.32$ respectively). Removal of the transplant glomerulopathy data eliminated the statistical significance between these groups, which indicated that the increased severity of chronic changes was dependent on the presence of transplant glomerulopathy in C4dpositive biopsy samples.

The researchers developed a scoring system to predict graft loss from histologic and clinical variables, in which 2 points are assigned for the presence of both transplant glomerulopathy and peritubular C4d immunostaining, while 1 point is allocated for transplant glomerulopathy or serum creatinine $>203 \mu \mathrm{mol} / \mathrm{l}$ alone. "The ... scoring system we described ... whereby a score of $\geq 3$ predicts rapid graft loss in $80 \%$ [of patients]," conclude the authors, "may be useful for clinicopathologic correlation and treatment of patients with long-term renal allografts."

Rowan Higgs

Original article Kieran, N. et al. Combination of peritubula C4d and transplant glomerulopathy predicts late renal allograft failure. J.Am. Soc. Nephrol. 20, 2260-2268 (2009) 